\title{
Comparative study of open and arthroscopic coracoid transfer for shoulder anterior instability (Latarjet)—clinical results at short term follow-up
}

\author{
Bartlomiej Kordasiewicz $^{1} \cdot \operatorname{Konrad}_{\text {Malachowski }}{ }^{1}$ - Maciej Kicinski ${ }^{1}$. \\ Slawomir Chaberek ${ }^{1} \cdot$ Stanislaw Pomianowski $^{1}$
}

Received: 9 August 2016 / Accepted: 11 December 2016/Published online: 30 December 2016

(C) The Author(s) 2016. This article is published with open access at Springerlink.com

\begin{abstract}
Purpose and hypothesis The aim of this study was to compare early clinical results after open and arthroscopic Latarjet stabilisation in anterior shoulder instability. Our hypothesis was the results of arthroscopic stabilisation were comparable with the results of open procedure.

Material and methods The clinical results of the patients after primary Latarjet procedure were analysed. Patients operated on between 2006 and 2011 using an open technique composed the OPEN group and patients operated on arthroscopically between 2011 and 2013 composed the ARTHRO group; 48 out of 55 shoulders $(87 \%)$ in OPEN and 62 out of 64 shoulders $(97 \%)$ in ARTHRO were available to follow-up. The average age at surgery was 28 years in OPEN and 26 years in ARTHRO. The mean follow-up was 54.2 months in OPEN and 23.4 months in ARTHRO. Intra-operative data were analysed regarding time of surgery, concomitant lesions and complications. Patient results were assessed with WalchDuplay, Rowe, VAS scores and subjective self-evaluation of satisfaction and shoulder function. Computed tomography scan evaluation was used to assess the graft healing.

Results Average time of surgery was significantly shorter in ARTHRO than OPEN: respectively 110 and 120 minutes. The number of intra-operative complications was six $(12.5 \%)$ in OPEN and five $(8.1 \%)$ in ARTHRO. The results were comparable in both groups, with no significant difference between OPEN and ARTHRO group: satisfaction rate $-96.8 \%$ and $91.9 \%$, shoulder function $-92.2 \%$ and $90 \%$, Walch-Duplay
\end{abstract}

Maciej Kicinski

keatech@msn.com

1 SPSK im. A. Grucy, Otwock, Trauma and Orthopedics Department, Centre of Postgraduate Medical Education, Warsaw, Poland score -83.9 and 76.7 respecively. A significant difference was reported in Rowe score: 87.8 in OPEN and 78.9 in ARTHRO. Another significant difference was found in the presence of "subjective apprehension"- a term referring to the subjective perception of instability with no signs of instability at clinical examination $-28.7 \%$ in OPEN and $50 \%$ in ARTHRO. Range of motion in both groups were comparable, however patients in OPEN had significantly lower loss of external rotation in adduction to the side comparing to the contralateral shoulder: $7^{\circ}$ versus $14^{\circ}$ in ARTHRO. Recurrence was reported in three cases in each group: $6.2 \%$ in OPEN and $4.8 \%$ in ARTHRO. A revision surgery was performed in four patients $(9.3 \%)$ in OPEN and six (9.7\%) in ARTHRO. Radiographic evaluation showed a significantly lower rate $(5 \%)$ of graft healing problems (fracture, non-union and osteolysis) after arthroscopic stabilisation, however a partial osteolysis of the proximal part of the bone block was significantly more frequent $(53.5 \%)$.

Conclusions The arthroscopic Latarjet stabilisation showed satisfactory and comparable results to open procedure. We recommend further investigation and development of arthroscopic technique.

Level of evidence: III

Keywords Arthroscopic Latarjet stabilisation · Coracoid transfer · Open Latarjet stabilisation · Shoulder instability

\section{Introduction}

Latarjet coracoid bone block stabilisation remains one surgical treatment option for anterior shoulder instability [1]. It also continues to be one of the most efficient stabilisation procedures. Bhatia et al. assessed the rate of recurrence following an open Latarjet procedure from 0 to $8 \%$ [2]. Walch reported a 
$1 \%$ recurrence rate which was amongst the best of results published [3]. In 2007 Lafosse described the arthroscopic technique of Latarjet stabilisation and in 2010, he reported the first 100 case results with $0 \%$ of recurrence [4]. From this moment onwards, the number of surgeons performing arthroscopic stabilisation has gradually increased and the discussion about superiority of one procedure or another has arisen. The goal of this study was to compare clinical results after open and arthroscopic Latarjet stabilisation. Our hypothesis was the result of arthroscopic Latarjet stabilisation, despite recently developed and slightly "experimental" technique are comparable with the results of open "gold standard" procedure.

\section{Material and methods}

In this study, the clinical results of two groups of the patients who underwent Latarjet procedure were analysed. All patients were operated on in the same institution by a senior author (B.K.). Only patients who underwent primary shoulder stabilisation were enrolled in the study, excluding all revision cases. Patients operated on between 2006 and 2011 using an open technique composed the first group (OPEN) and patients operated on between 2011 and 2013 using an arthroscopic stabilisation composed the second group (ARTHRO). In 2011 the surgeon performing the procedures abandoned open stabilisation, and since then began using only arthroscopic techniques, so there was no patient randomisation. In 2014 all patients operated on were invited for a control review: clinical examination with standard X-ray and CT scan evaluation. Informed consent was obtained from all individuals included in the study - patients were informed about potential risk of CT - one patient in each group refused participation. Additionally all data concerning surgical procedures (time, intra-operative findings and complications) were retrieved from the hospital database. This case-control study with clinical and radiological follow-up was granted approval of the institution's ethical committee (Ethical Board of the Postgraduate Medical Education Centre, nr 38/PB/2014). Clinical assessment was performed by two senior residents, not involved in surgery. Group characteristics were presented in Table 1. Patient results were assessed with Walch-Duplay and Rowe scores and pain in VAS [5, 6, 34]. Patients also assessed satisfaction and function answering two questions (rating from 0 to $100 \%$ ): "How satisfied are you with the surgery outcome?" and "How do you judge your shoulder function in comparison to activity before the first episode of instability?" $100 \%$ being the highest mark and $0 \%$ the lowest. Also, a particular term has been forged and used in the study: "subjective apprehension" referring to a patient's subjective perception of shoulder instability, whereas no signs of instability are revealed at clinical examination. Radiological evaluation was supervised by the senior specialist in a musculoskeletal radiology. The radiological results were blinded to the clinical outcome. The CT scans were performed on the GE Bright Speed 16-row scanner, using the standard shoulder protocol and the slice thickness $0.63 \mathrm{~mm}$. All measurements were made using the Carestream software version 11.4 (Carestream Health; Rochester, NY, image processing software). $3 \mathrm{D}$ and multiplanar reformations were used for the best visualisation of the anatomy and the screws. Graft fusion was determined by the presence of the bone bridge between the coracoid and the glenoid. In case of non-union it was identified in two possible options: stable - with no lysis around the screws and unstable - with hardware loosening and graft dissociation. Bone block osteolysis was evaluated in both axial and sagittal plane and described as total-concerning the entire graft or partial - around the superior or the inferior screw. Other radiological data are not to be discussed in this paper, as another manuscript is being dedicated for this vast subject analysis (Part II of the study). All statistical analysis was performed using the STATISTICA 7.0 software (StatSoft, Inc., STATISTICA for Windows, Tulsa, OK). The analysed groups were compared using descriptive statistics and nonparametric
Table 1 OPEN and ARTHRO group pre-operative characteristics, ns- statistically insignificant $(p<0.05$ considered statistically significant)

\begin{tabular}{llll}
\hline & Open & Artrho & $\mathrm{p}$ \\
\hline F/M & $\mathbf{2 / 4 5 ( 4 . 2 \% / 9 5 . 8 \% )}$ & $\mathbf{7 / 5 5}(\mathbf{1 1 . 3 \%} / \mathbf{8 8 . 7 \%})$ & $\mathbf{P}<\mathbf{0 . 0 5}$ \\
Age at 1st episode & $23.8(16-56)$ & $21(13-40)$ & $\mathrm{ns}$ \\
Age at surgery & $28(18-59)$ & $26(16-44)$ & $\mathrm{ns}$ \\
Time to surgery (months) & $49.9(6-180)$ & $57.5(4-228)$ & $\mathrm{ns}$ \\
No. dislocations & $5(0-30)$ & $5(0-40)$ & $\mathrm{ns}$ \\
No. subluxations & $15(0-80)$ & $10(0-50)$ & $\mathrm{ns}$ \\
Dominant/non-dominant & $33 / 15(68.7 \% / 31.3 \%)$ & $37 / 24(59.7 \% / 38.7 \%)$ & $\mathrm{ns}$ \\
& & 1 bilateral & $\mathrm{ns}$ \\
Pain in live activity & $16(33.3 \%)$ & $13(20.97 \%)$ & $\mathbf{P}<\mathbf{0 . 0 5}$ \\
Laxity & $\mathbf{2 3 ( 4 7 . 9 \% )}$ & $\mathbf{3 8}(\mathbf{6 1 . 3 \%})$ & $\mathrm{ns}$ \\
Walch-Duplay & $15(-10$ to 25$)$ & $20(-10$ to 25$)$ & $\mathrm{ns}$ \\
Rowe score & $25(15$ to 30$)$ & $27(0$ to 30$)$ & \\
\hline
\end{tabular}


statistics analysis. Cross tabulation tables were used for the descriptive statistics, as for nonparametric statistics the following tests were used: Mann-Whitney U test, Kolmogorov Smirnov test and Friedman ANOVA. Spearman rank R correlation (Spearman - R) was used to assess the relationship between variables. A $p$-value of $<0.05$ was considered statistically significant.

\section{Surgical technique}

The decision to perform Latarjet procedure was based on a patient's medical history, clinical examination and radiological findings. Routinely all patients underwent X-ray examination (AP and Y view) and additionally CT or MRI scans. Patients that qualified for Latarjet stabilisation were supposed to have several risk factors, usually in combination: professional sport or high risk activity, Hill-Sachs lesion of more than $15 \%$ of humeral head diameter, glenoid bone loss $>$ $10 \%$, laxity (thumb-forearm distance less than $2 \mathrm{~cm}$, external rotation in adduction $>85$ degrees). In addition, in the arthroscopic group, the final decision on Latarjet stabilisation was undertaken second to a glenohumeral joint inspection in regard to anterior soft tissue quality and an engagement of HillSachs lesion: anterior glenoid rim and Hill-Sachs lesion contact or dislocation in an "apprehention position" of $90^{\circ}$ of abduction and external rotation, according to "on track/off track" hypothesis [7]. All open procedures were performed according to a technique described by Walch [8], whereas all arthroscopic procedure according to Lafosse's last modification technique, using specific arthroscopic instruments (DePuy, Mitek, Johnson\& Johnson) [4, 9], in beach chair position under general anesthesia and interscalene block (Fig. 1).

Post-operative protocol was the same after open and arthroscopic stabilisation: a simple sling to prevent pain for three to ten days after surgery depending on patient's pain control. In this period, active exercise of fingers, wrist and elbow were introduced along with the passive, pendulum exercises of the shoulder. As soon as the pain and the post-operative swelling decreased, the sling was discontinued and active mobility was started, always within pain free limits and respecting natural scapulo-thoracic rhythm. Water exercises were recommended after wounds healed up. After two to four weeks stretching exercises were introduced and second to a full forward flexion restoration muscle strengthening exercises were started, no sooner than eight weeks after the surgery. Contact sports were allowed after restoration of a full range of motion and strengthening exercises, but no sooner than three months after surgery.

\section{Results}

In OPEN group 48 out of 55 shoulders $(87 \%)$ were available to follow-up. Seven patients were lost to follow-up, four refused participation (only phone interview). Forty-four shoulders had post-operative X-ray and 43 had CT scan (78.2\%) - one patient refused CT evaluation. In ARTHRO group 62 out of 64 shoulders $(97 \%)$ were available to follow-up and 61 patients were available to radiological evaluation (96.8\%) — one patient did not accept radiological part of the study. Patient characteristics were presented in Table 1. Both groups were relatively homogenous as only significant differences $(p<0.05)$ were: female/male ratio and laxity. The most important difference remained follow-up: 54.2 months in OPEN and 23.4 months in ARTHRO $(\mathrm{p}<0.05)$.

\section{Operative data and complications}

The average time of surgery was significantly shorter $(\mathrm{p}<0.05)$ for ARTHRO group-Table 2. It is important to notice the presence of technical difficulties in the first open procedures - the first procedure lasted 190 minutes and was the longest in this series, with the reduction to approximately 60 minutes in the later cases. The average time was influenced by the complications related to the graft fracture that made graft fixation more difficult and time consuming. Our first arthroscopic stabilisation took 210 minutes with reduction to 70 minutes during the latest procedures.

In regards to intra-operative findings there was a statistically significant $(\mathrm{p}<0.05)$ difference in number of patients without intra-operatively confirmed bone loss. Concomitant injuries were found in five patients $(10.4 \%)$ in OPEN: three rotator cuff lesions: two supraspinatus and one superior subscapularis tendon, one loose body and one cartilage loss of $1 / 3$ anterior of glenoid (four in five patients had injuries repaired) and in six patients $(9.7 \%)$ in ARTHRO group: one partial supraspinatus tear, two SLAP lesions, one SLAP lesion with loose bodies, one loose body, one posterior labrum tear (all patients received successful repair of concomitant injuries). In ARTHRO group the lesions were predominantly intra-articular (SLAP, posterior labrum) being less likely to be detected and treated in an open procedure; however, this remained statistically insignificant and did not correlate with the clinical results. In OPEN group there were five graft fractures $(10.4 \%)$ : two of these cases occurred during the coracoid osteotomy and another three cases while fixing the graft. In two of these patients it was a longitudinal outer cortex infraction that remained stable-in one case a final fixation was peformed with one screw only. In one case the proximal part of the graft was fractured whilst coracoid osteotomy and graft fixation was achieved with one distal screw. In another two cases the longitudinal fracture line was complete - the graft was "reconstructed"-wrapped around with sutures and fixed with one screw in one patient and with two screws and an anchor-to suture the conjoined tendon to the glenoid to release the traction forces from the graft and to enhence the tenodezis effect - in another patient. All these patiens were satisfied with the results: satisfaction 

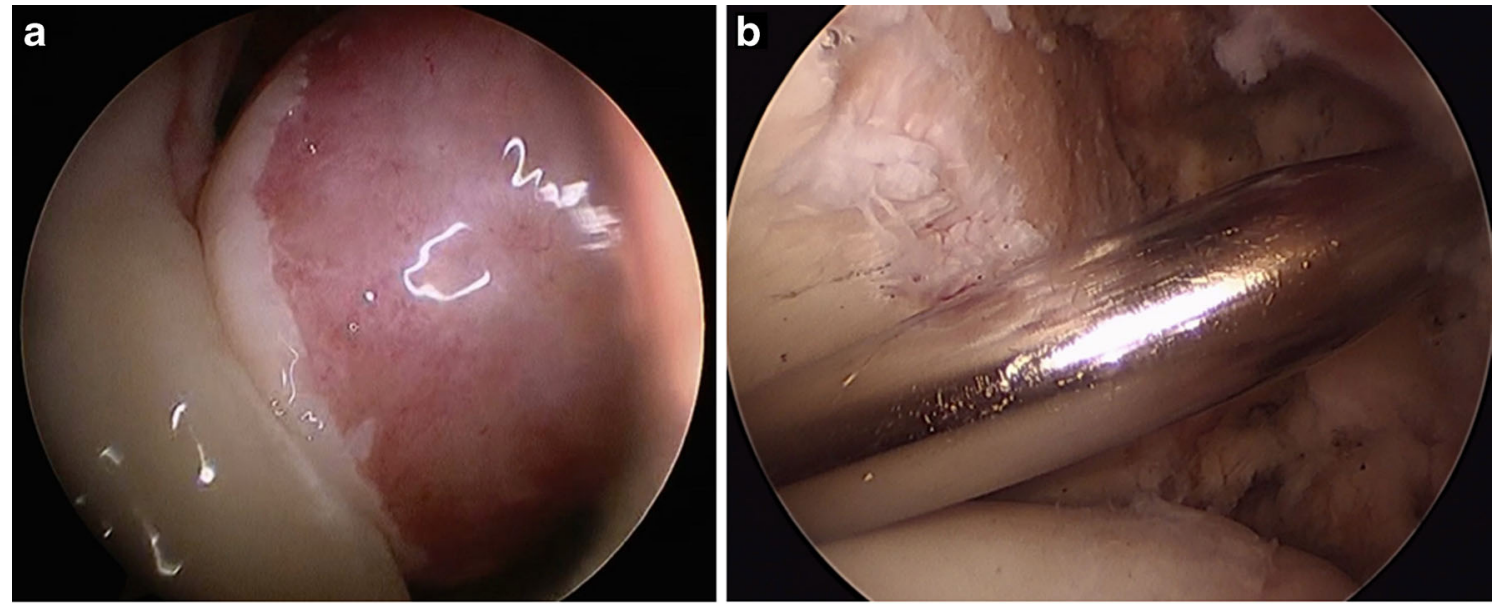

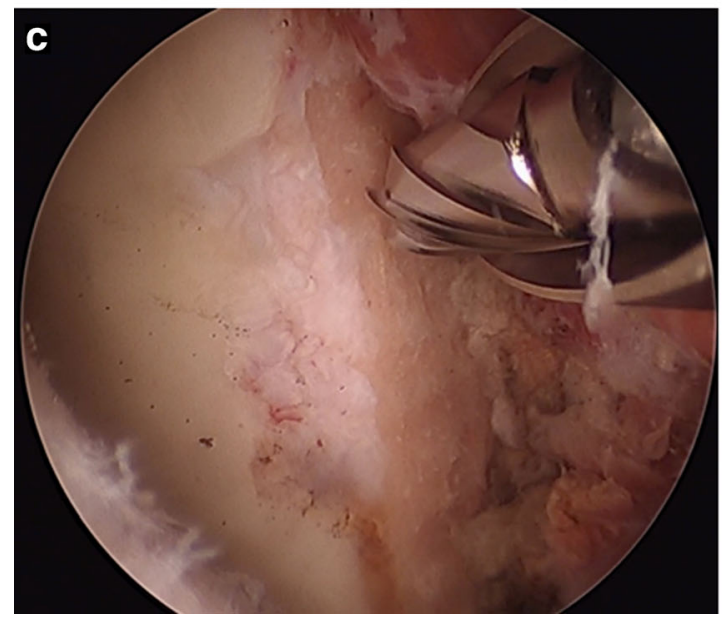

Fig. 1 A few steps of the arthroscopic Latarjet procedure (right shoulder): a) Hill-Sachs lesion appreciation and its "engaging" - this test is performed in "apprehention position" of 90 degrees of abduction and external rotation, according to "on track/off track" hypothesis (posterior portal view); b) Subscapularis split - a swithing stick is introduced from posterior portal at the level of glenoid bone loss, usually at the junction of its inferior third and superior two-thirds of the subscapularis muscle (anterolateral portal view); c) Final glenoid neck preparation - after subscapularis split a bur is used to create a flat bed

80-100\%, Rowe score 70-100, Walch-Duplay score 70-100, with no recurrence. One of these five patients refused participation in CT evaluation (100\% satisfaction, 100 points in the scores). Two out of these four patients had graft osteolysis on $\mathrm{CT}$, and two grafts were healed (Fig. 2). One case (2.1\%) of complication related to hardware malposition was found - the conflict of a screw and an overlaping graft with a humeral head. The screws were removed and the graft trimmed six months after the initial surgery. This patient reported good subjective results; despite the fact he developed a moderate glenohumeral joint arthrosis and clinically achieved 70 points in Walch-Duplay and 80 in Rowe score. Another patient (2.1\%) had superficial skin abrasion due to retractor positioning, with an excellent result on the final clinical examination. In ARTHRO group there were five intraoperative complications: one medial cutaneous antebrachial nerve injury (with

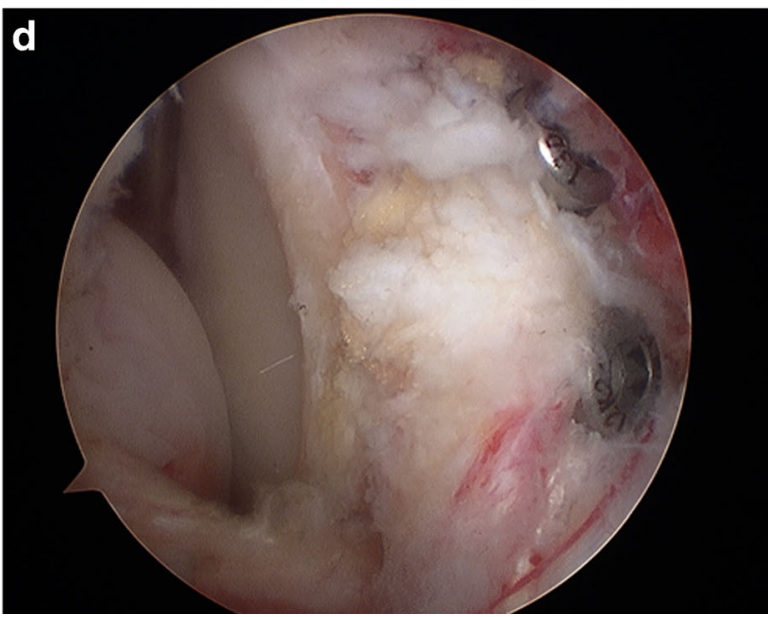

of bleeding bone (anterolateral inferior portal view); d) Coracoid final fixation onto scapular neck - the harvested coracoid was introduced through the subscapularis split onto glenoid neck between 3 and 5 o'clock position, 1 to $2 \mathrm{~mm}$ medial to the glenoid rim to avoid graft lateralisation - temporary fixation was achieved using long quide wires - when the graft placement was optimal, $3.2 \mathrm{~mm}$ cannulated drill was used and a final fixation was completed with $3.5 \mathrm{~mm}$ cannulated screws. Drilling and fixation were performed through the double-barrel cannula from medial portal (anterolateral inferior portal view)

Table 2 OPEN and ARTHRO group intraoperative data, nsstatistically insignificant $(\mathrm{p}<0.05$ considered statistically significant)

\begin{tabular}{llll}
\hline & Open & Arthro & $\mathrm{p}$ \\
\hline Time of surgery & $\mathbf{1 2 0}(\mathbf{6 0 - 1 9 0 )}$ & $\mathbf{1 1 0}(\mathbf{7 0 - 2 1 0 )}$ & $\mathbf{p}<\mathbf{0 . 5}$ \\
$\begin{array}{l}\text { Intraoperative complications } \\
\text { Lateral graft position-graft }\end{array}$ & $6(12.5 \%)$ & $5(8.1 \%)$ & $\mathrm{ns}$ \\
$\quad$ trimmed & $5(10.4 \%)$ & $3(4.8 \%)$ & $\mathrm{ns}$ \\
$\begin{array}{l}\text { Concomitant injuries } \\
\text { Repaired injuries }\end{array}$ & $4(8.3 \%)$ & $6(9.7 \%)$ & $\mathrm{ns}$ \\
No glenoid bone loss & $\mathbf{0 ( 0 \% )}$ & $\mathbf{1 7}(\mathbf{2 7 . 4 \% )})$ & $\mathbf{p}<\mathbf{0 . 5}$ \\
Fixation other than 2 screws & $4(8.3 \%)$ & $1(1.6 \%)$ & $\mathrm{ns}$ \\
Screw I in mm (most often) & $30-44(40)$ & $32-42(36)$ & $\mathrm{ns}$ \\
Screw II in mm (most often) & $30-42(40)$ & $32-44(38)$ & $\mathrm{ns}$ \\
\hline
\end{tabular}


excellent clinical follow-up); one broken graft at the level of proximal hole, fixed with one screw; one superior screw fixed too deep in the graft due to poor bone quality; two distal cortical destroyed whilst drilling distal hole in the graft-no top hats (washers) for fixation. In the last three patients no compression was achieved with only antirotational effect of the second screw. Four out of five complications (6.4\%) concerned coracoid drilling or fixation. One patient with only one screw fixation had a dislocation seven months after surgery and one patient with only antirotational distal screw fixation had a subluxation two weeks after surgery - these two patients had revision surgery and were considered as a failure. Two out of four patients with "graft problems" presented excellent results on clinical and CT follow-up (Fig. 3).

Intra-operatively overlaping graft required some trimming at the lateral border in two cases $(4.2 \%)$ in OPEN and three (4.8\%) in ARTHRO groups. In general we found no influence of intra-articular lesions and intraoperative complications on the final results, except the subjective evaluation of satisfaction in OPEN group: the patients with associated lesions rated it up to $83 \%$, as ones without $93.4 \%(\mathrm{p}<0.05)$.

\section{Post-operative results}

The results are presented in Table 3. The only statistically significant difference was in Rowe score: 87.8 in OPEN and 78.9 in ARTHRO group $(\mathrm{p}<0.05)$ and the "subjective apprehension": $28.7 \%$ in OPEN and 50\% in ARTHRO $(\mathrm{p}<0.05)$, which was neither confirmed in clinical examination nor patients' satisfaction. Patients' range of motion in both groups were very similar (Table 4), minimally better in OPEN than in ARTHRO group. However, patients in OPEN group had a significantly lower loss of external rotation with the arm at side (delta ER1) when compared to contralateral
Fig. 2 Graft healing in patients with intra-operative bone block fracture - open stabilisation with one screw fixation. $\mathbf{a}, \mathbf{b}-$ healed graft; c,d - graft osteolysis
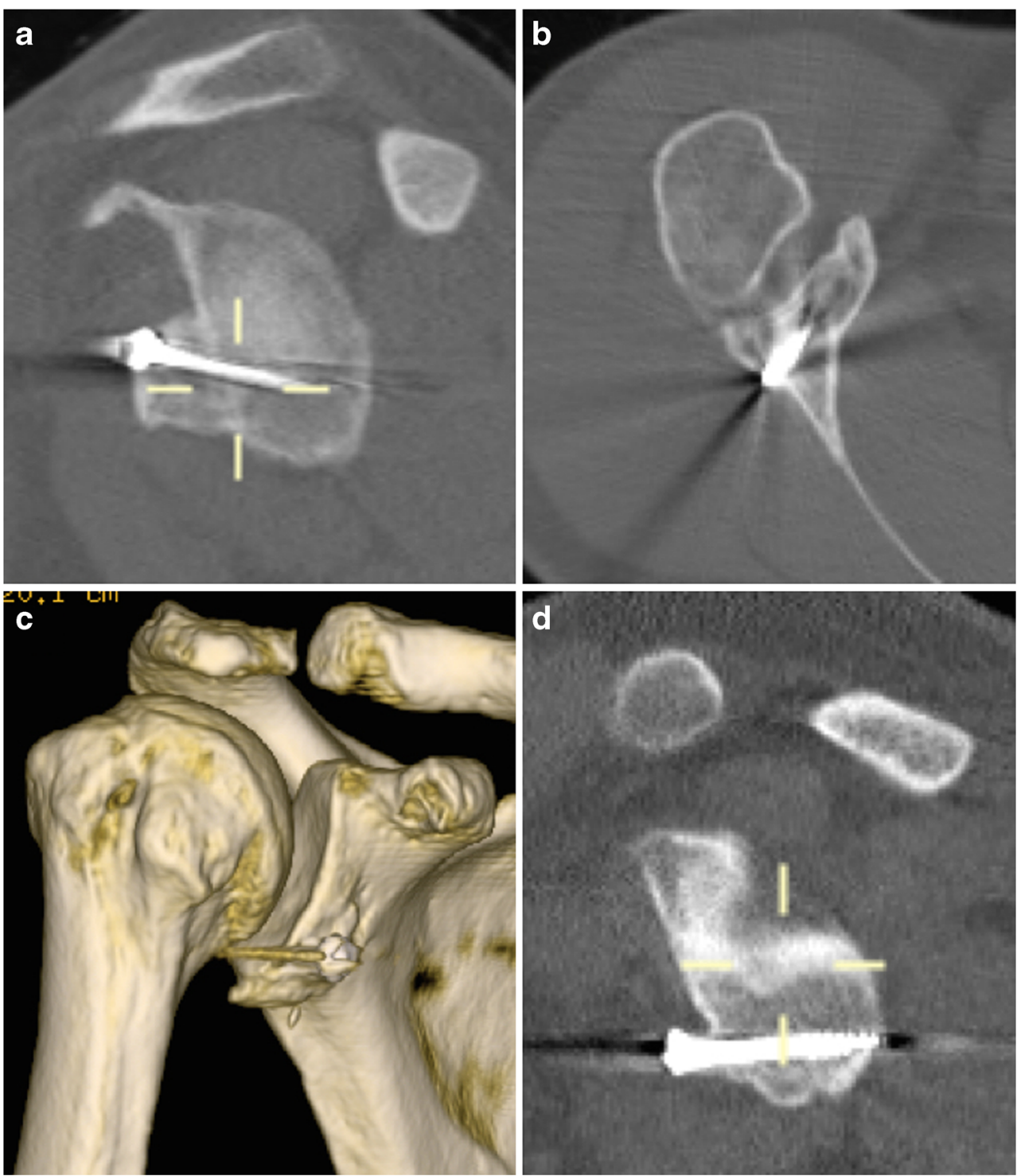
Fig. 3 Graft position in patients with intra-operative complication - distal hole in the coracoid was drilled to close to the coracoid tip with distal cortical destruction - it diminished the compression possibility - in this case the distal screw played mainly antirotational effect. $\mathbf{a}, \mathbf{b}$ - graft and screw fracture - subluxation episode, $\mathbf{c}, \mathbf{d}$ - graft healed in proper position; lysis of the proximal part of the graft
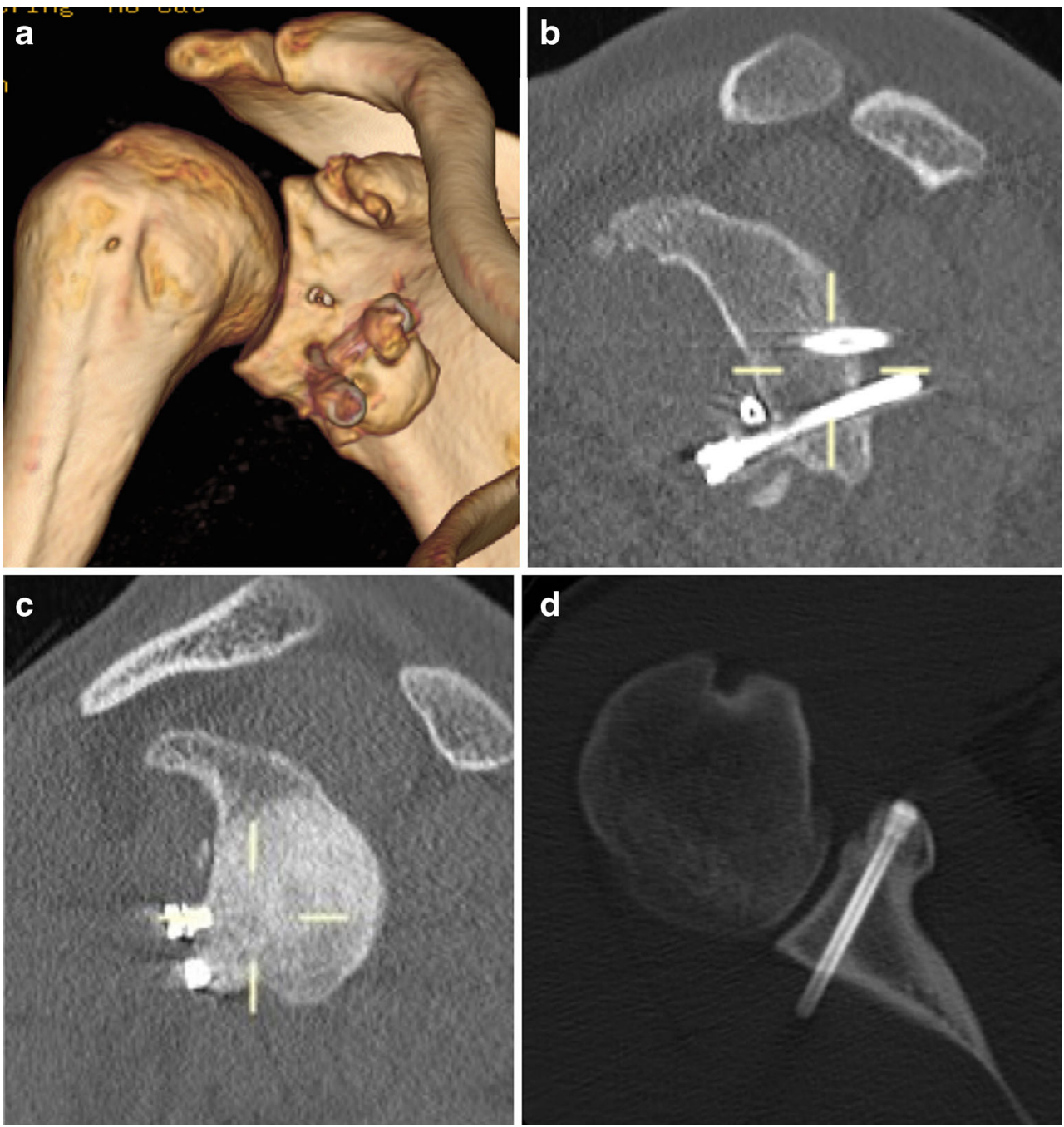

shoulder: $7^{\circ}$ in OPEN and $14^{\circ}$ in ARTHRO group $(\mathrm{p}<0.05)$. Interestingly loss of external rotation in $90^{\circ}$ of abduction (delta ER2) was comparable: $5.6^{\circ}$ in OPEN and $6.2^{\circ}$ in ARTHRO group. There was a significant $(\mathrm{p}<0.05)$ influence of two parameters on the final results: subjective apprehension and loss of external rotation. In ARTHRO group it was significantly correlated with function, Walch-Duplay and Rowe scores. In other words, the more "subjective apprehension" patients reported and the more loss of external rotation they had, the worse the achieved results were. It was also interesting to find the correlation between the time of follow-up and the clinical results (scores, satisfaction and function), which suggested that patient satisfaction improved with time.

\section{Recurrence}

There were three cases of recurrence in each group, which represented $6.2 \%$ in OPEN and $4.8 \%$ in ARTHRO group - a difference statistically insignificant. In OPEN group there were two dislocations and one subluxation that appeared respectively eight, nine and seven months after surgery. Two patients were revised with iliac crest bone block and one with conjoined tendon and fractured graft refixation with interference screw-in the last case the patient had severe trauma

Table 3 OPEN and ARTHRO group clinical results and complications, ns- statistically insignificant $(\mathrm{p}<0.05$ considered statistically significant)

\begin{tabular}{llll}
\hline & Open & Arthro & $\mathrm{p}<0.05$ \\
\hline Follow-up (months) & $\mathbf{5 4 . 2 ( 3 0 - 8 1 )}$ & $\mathbf{2 3 . 4 ( 1 3 - 4 4 )}$ & $\mathbf{p}<\mathbf{0 . 0 5}$ \\
Walch - Duplay score & $83.9(10-100)$ & $76.7(0-100)$ & $\mathrm{ns}$ \\
Rowe score & $\mathbf{8 7 . 8 ( 2 5 - 1 0 0 )}$ & $\mathbf{7 8 . 9}(\mathbf{1 5 - 1 0 0 )}$ & $\mathbf{p}<\mathbf{0 . 0 5}$ \\
VAS & $0.77(0-5)$ & $1.38(0-8)$ & $\mathrm{ns}$ \\
Satisfaction \% & $96.8(60-100)$ & $91.9(40-100)$ & $\mathrm{ns}$ \\
Function \% & $92.2(40-100)$ & $90(30-100)$ & $\mathrm{ns}$ \\
Subjective apprehension & $\mathbf{1 3 ( 2 8 . 7 \% )}$ & $\mathbf{3 1 ( 5 0 \% )}$ & $\mathbf{p}<\mathbf{0 . 0 5}$ \\
Recurrence & $3(6.2 \%)$ & $3(4.8 \%)$ & $\mathrm{ns}$ \\
Revisions & $4(8.3 \%)$ & $8(12.9 \%)$ & $\mathrm{ns}$ \\
No discomfort & $33(68.7 \%)$ & $33(55 \%)$ & $\mathrm{ns}$ \\
\hline
\end{tabular}


Table 4 OPEN and ARTHRO group clinical results in terms of range of motion, ns- statistically insignificant $(\mathrm{p}<0.05$ considered statistically significant)

\begin{tabular}{llll}
\hline & Open & Arthro & $\mathrm{p}<0.05$ \\
\hline flexion & $179(160-180)$ & $177(140-180)$ & $\mathrm{ns}$ \\
abduction & $177(140-180)$ & $176(70-180)$ & $\mathrm{ns}$ \\
ER1 & $61.7(0-85)$ & $59(10-90)$ & $\mathrm{ns}$ \\
delta ER1 & $-\mathbf{7}(-\mathbf{5 0}$ to $\mathbf{1 5})$ & $-\mathbf{1 4}(-\mathbf{7 0}$ to 30) & $\mathbf{p}<\mathbf{0 . 0 5}$ \\
$\begin{array}{l}\text { delta IR1(spinal } \\
\text { processes) }\end{array}$ & $-1.2(-10$ to 2$)$ & $-1.2(-10$ to 0$)$ & $\mathrm{ns}$ \\
ER2 & $79(60-95)$ & $81.4(30-95)$ & $\mathrm{ns}$ \\
delta ER2 & $-5.6(-30$ to 10$)$ & $-6.2(-60$ to 10$)$ & $\mathrm{ns}$ \\
\hline
\end{tabular}

ER1- external rotation in adduction. ER2- external rotation in abduction. IR1-internal rotation

with a fresh graft fracture. In ARTHRO group there were two subluxations and one dislocation occurring two weeks (unknown mechanism of trauma with a screw break), 18 months (severe trauma with subluxation and superior screw break with fully healed graft) and seven months (dislocation with a screw break after single screw fixation) respectively. All these patients were revised: two had an iliac crest bone graft and one with a healed bone block had a remplissage procedure.

\section{Revisions}

In OPEN group there were four revisions (8.3\%): three for recurrence and one due to the conflict of a screw and an overlaping graft with a humeral head. In this last case the screws were removed and the graft trimmed six months after the surgery. This patient reported good subjective results; despite the fact he developed a moderate glenohumeral joint arthrosis and clinically achieved 70 points in Walch-Duplay and 80 in Rowe scores. In ARTHRO group there were six revisions (9.7\%): three (4.8\%) due to recurrence; one patient had superior pole of the graft osteolysis and screw loosening - the screw was removed; one had a frozen shoulder suffering from lack of external rotation - he underwent arthroscopic arthrolysis 26 months after the initial surgery; one patient due to reasons not related with primary surgery - a car accident with a posterior shoulder subluxation and a posterior labrum injury - underwent posterior labrum repair 24 months after initial stabilisation. Another two cases were not operated on so far, due to patient hesitation, but revision surgery shall be taken under consideration for a discomfort related probably to dorsal screw protrusion irritating the infraspinatus muscle. If these two potential patients were added we would come up with eight revisions (12.9\%), which still would be insignificantly higher than in OPEN group. In ARTHRO group these cases could be divided into revisions related to recurrence (4.8\%), hardware problems (4.8\% including planned revision due to too long screws), stiffness (1.6\%) and other pathologies not related with prior surgery $(1.6 \%)$.

\section{Radiographic results}

An early graft fracture was reported in two cases (3.3\%) in ARTHRO in two of three patients with recurrence. There was one case $(2.4 \%)$ of the graft fracture in OPEN group in the patient above described with recurrence and conjoined tendon refixation. The non-union rate was significantly higher $(\mathrm{p}<0.05)$ in OPEN than in ARTHRO group: five $(11.9 \%)$ versus one $(1.7 \%)$. Two patients in OPEN presented the unstable non-union with graft and hardware dissociation. Nevertheless these two patients remained stable, with high and moderate satisfaction (Walch-Duplay, Rowe score and satisfaction were respectively 100,100 and $100 \%$ in one patient and 70, 90 and 65\% in the second one). The stable nonunion was found in three cases (7.1\%) in OPEN group: versus one case in ARTHRO group (1.7\%) - all these cases were stable with good clinical results.

The entire graft osteolysis was significantly higher $(\mathrm{p}<0.05)$ in OPEN group-five cases (11.9\%) versus 0 in ARTHRO. Two of them were the sequel of the intra-operative complication - graft fracture. Three of these five cases presented satisfactory results: Walch-Duplay score 80 to 100, Rowe score 95 to 100 and satisfaction $100 \%$. Another two of these five patients (not related to the intra-operative complication) had recurrence. All mentioned above graft healing problems together (fractures, nonunion and osteolysis) were significant $(\mathrm{p}<0.05)$ more often in OPEN than in ARTHRO group: respectively 11 (26.2\%) and $3(5 \%)$. The opposite results were found concerning partial graft osteolysis - a significantly higher rate of the partial graft osteolysis at the level of the superior screw was reported in ARTHRO group - 32 patients $(53.3 \%)$ versus ten patients $(23.8 \%)$ in OPEN group. Osteolysis at the level of the inferior screw was noted only in one case in OPEN group (2.4\%) and two cases in ARTHRO (3.3\%). In ARTHRO group a screw fracture was reported in three cases $(5 \%)$-all patients with recurrence. No screw fracture was reported in OPEN group. This difference did not reach statistical significance. Only two correlations statistically significant $(p<0.05)$ were reported with the above results. In ARTHRO group the correlation was found between postoperative graft fracture and worse clinical evaluation in Walch-Duplay and Rowe scores. In OPEN group we found the correlation between graft osteolysis and discomfort reported in patients activity.

\section{Discussion}

The methodology of this study is not free of certain weaknesses, which shall be taken into consideration while analysing it: 
1. Both groups have a different follow-up, which might raise suspicion it influenced the patient's outcome. However, Griesser in his study reported that $73 \%$ of recurrence occurred within the first 12 months after surgery [10]. In addition, in our observation, most of the parameters assessed remained unchanged after 12 months after the surgery, with exception of a subjective rating of the shoulder's function and apprehension, which may improve in time.

2. As it was not a planned prospective study, these two groups had a significantly different number of patients with laxity.

3. The pre-operative radiologic data were not collected in a systematic and repetitive manner so we decided not to include it into the study. This is also a reason why the radiographic pre-operative bone loss was not assessed in patient data.

4. The clinical results of patients did not include the time of recovery and rehabilitation to restore full activity, which shall be a very important aspect comparing open and arthroscopic technique.

5. The experience and technical skills of the surgeon could strongly influence the results, mainly in OPEN group. It is important to mention that the surgeon started his learning curve of both techniques from the very beginning. One might say that five years later the surgeon was more experienced and could learn new techniques more easily, which might have resulted in time of surgery shortening and in the number of intra-operative complications decreasing. It is to emphasise that all techniques require some learning curve. This "learning curve" was probably an important factor diminishing the value of this study. Nevertheless this study has some strong points. The advantage was the fact that all patients were operated on by a single surgeon. We believe it is still interesting to compare these two techniques and the results from the starting point. Another important point is a fact that this is, to our knowledge, one of the few studies published comparing these two techniques. The first study comparing open and arthroscopic stabilisation results was Cunningham's et al. report evaluating 28 patients in the arthroscopic group and 36 in the open one [11]. A significantly higher operative time in the arthroscopic stabilisation versus open was reported: 146 and 81 minutes respectively. It was also shown that surgical time came close to that of open stabilisation after 20 procedures. Cunningham et al. reported no dislocation after open and one $(3,6 \%)$ after arthroscopic procedure. No intra-operative complications were reported in either group but there were significantly more postoperative complications in the arthroscopic group: $29 \%$ and $11 \%$ respectively. In arthroscopic group there were two non-unions, 3 migrations, one infection, one haematoma and one recurrence. In open group there were two infections and two haematomas. Additionally conversion from arthroscopic to open technique was necessary in three cases. One patient with recurrency (3.6\%) after arthroscopic technique required restabilisation. Similar external rotation was found in both groups: $55^{\circ}$ in the arthroscopic group and $51^{\circ}$ in the open. More results reported in the literature concerning open or arthroscopic techniques were compared in Tables 5, 6 and 7. Our study results are relatively comparable to those reported in up to date literature. Several important aspects shall be discussed more meticulously.

\section{Complications, recurrence and revisions}

We found the percentage of intra-operative complications was comparable, with no significant differences: $12.5 \%$ in OPEN versus $8.1 \%$ in ARTHRO. In OPEN group five cases were complicated with a graft fracture (10.4\%) and in ARTHRO group four complications (6.4\%) concerned bone graft drilling or fixation. We found no statistical correlation between the intra-operative graft fractures or any fixation problems and clinical and radiological results. However, it could be noticed that two of the three recurrences in ARTHRO group had some graft fixation related problems. Additionally all three cases of recurrence in ARTHRO had a screw fracture. This fact did not reach any statistical significance, so this could not be treated as any conclusion, however it could be only our suspicion that combination of technical fault and cannulated screws could increase the risk of graft fracture. Shah et al. reported using canulated screws as a risk factor [16]. Three cases of recurrency in each group constituted $6.2 \%$ in OPEN and
Table 5 Summary data of the open and arthroscopic Latarjet procedure published in the literature

\begin{tabular}{lll}
\hline Variable & Arthroscopic & Open \\
\hline Concomitant lesions & $7 \%[4]-13 \%[12]$ & $3.4 \%[13]-11.6 \%[2]$ \\
Time of surgery (in minutes) & $45[4]-240[4]$ & $45^{*}-81[11]$ \\
Recurrence & $0 \%[4]-3.6 \%[11]$ & $0 \%[13]-10 \%[14]$ \\
Complications & $10 \%[12]-29 \%[11]$ & $4.9 \%[15]-25 \%[16]$ \\
Loss of external rotation in adduction & $17^{\circ}[17]-18^{\circ}[4]$ & $5.1^{\circ}[15]-10.7^{\circ}[18]$ \\
Revisions & $0 \%[19]-15.6 \%[20]$ & $3.4 \%[18]-7 \%[10]$ \\
\hline
\end{tabular}

* time of surgery reported during live session whilst Annecy Live Surgery Course 2009-2015 
Table 6 Detailed summary of the open Latarjet procedure results published in the literature

\begin{tabular}{|c|c|c|c|c|c|c|c|}
\hline & Complications & Revisions & Recurrence & ER loss & Scores & Return to sport & FU \\
\hline Allain et al.[13] & $7 \%$ & $4 \%$ & $0 \%$ & $21^{\circ} \mathrm{ER} 2$ & $84 \mathrm{C}$ & $80 \%$ & 172 \\
\hline Burkhart et al.[15] & $4.9 \%$ & $1 \%$ & $4.9 \%$ & $5.1^{\circ} \mathrm{ER} 1$ & $\begin{array}{l}91.7 \mathrm{~W}-\mathrm{D} \\
94.4 \mathrm{C}\end{array}$ & $96.4 \%$ & 59 \\
\hline Cerciello et al.[21] & & & $2.2 \%$ & & 89.3 W-D & $96.3 \%$ & 85 \\
\hline Mahirogullari et al.[14] & $16.6 \%$ & $13.3 \%$ & $10 \%$ & $4^{\circ} \mathrm{ER}$ & $88.1 \mathrm{R}$ & & 28 \\
\hline Mizuno et al.[22] & & $1.5 \%$ & $5.9 \%$ & & $\begin{array}{l}89.6 \mathrm{R} \\
90.9 \% \mathrm{SSV}\end{array}$ & $93.4 \%$ & 240 \\
\hline Hovelius et al. [18] & $1.7 \%$ & $3.4 \%$ & $13.4 \%$ & $\begin{array}{l}10.7^{\circ} \text { ER1 } \\
12.4^{\circ} \text { ER2 }\end{array}$ & $89.4 \mathrm{R}$ & $86 \%$ & 180 \\
\hline Shah et al.[16] & $25 \%$ & 14.6 & $8 \%$ & $10.3^{\circ} \mathrm{ER}$ & $\begin{array}{l}78 \% \mathrm{R} \\
81.5 \% \mathrm{SSV}\end{array}$ & & 9.4 \\
\hline
\end{tabular}

ER1- external rotation loss in adduction, ER2- external rotation loss in abduction; R-Rowe, C- constant, W-D Walch-Duplay, SSV- subjective shoulder value; FU- follow-up (in months)

$4.8 \%$ in ARTHRO. In ARTHRO it was slightly more than reported so far by other authors performing arthroscopic techniques (Table 7), but still remained comparable to the data of an open technique: ranging from $0 \%-1.7 \%[13,23]$ to $8-9 \%$ $[14,16]$. Bessiere et al. reported $12 \%$ recurrence rate after an open procedure: five of six cases were related with technical faults [24]. In OPEN group two of three cases were related with the graft osteolysis and one with traumatic graft fracture but these facts remained statistically insignificant. The rate of complications in our study remain similar to the results already published, ranging from $10 \%$ to about $30 \%$ in both techniques. We reported no severe complications such as vascular impairment, motor nerve palsy or infection, which are very rare; however, we found in the literature that Shah et al. reported $10 \%$ (five patients) of neurological complications in open technique: three cases of temporary nerve palsies: two musculocutaneus and one radial nerve-fully recovered in time; two persistent axillary palsies including one sensory and one partial motor deficit [16]. The number of revisions in ARTHRO group (12.9\%) stays comparable to the results published in the literature (Table 7). In OPEN group the rate of revisions $(8.3 \%)$ seems to be higher then reported in other studies - it may be related with discussed above surgeon's learning curve, however it was not confirmed statistically in this study. Butt and Charalambous in their review study regarding open technique reported $4.9 \%$ of revision, $6 \%$ of recurrence and $6.5 \%$ of hardware problems [25].

\section{Clinical results}

Most of the results were slightly better in OPEN group, which was statistically insignificant, except for three parameters: a loss of external rotation in adduction to the side, subjective apprehension and Rowe score. Subjective apprehension, understood as a fear to return to overhead activities, might be some form of "micro-instability" - due to a biomechanic problem that we cannot assess or it could represent some mutli-directional instability related to a patient laxity, that occurred significantly more frequent in ARTHRO group patients. Collin et al. showed the rate of persisting apprehension of $34 \%$ after the open Latarjet procedure, whereas the recurrence rate was only $5 \%$. He recommended performing an additional capsuloplasty in patients with preoperative hyperlaxity [26]. It is also possible that subjective apprehension is related with an arthroscopic technique: excision of the capsule and MGHL could create some proprioceptive deficit related to the lack of some mechanoreceptors [27, 28]. We also think it might be a psychologically originated effect, as none

Table 7 Detailed summary of the arthroscopic Latarjet procedure results published in the literature

\begin{tabular}{|c|c|c|c|c|c|c|c|}
\hline & Complications & Revisions & Recurrence & ER loss & Scores & Return to sport & $\mathrm{FU}$ \\
\hline Dumont et al.[20] & $7.8 \%$ & $15.6 \%$ & $1.6 \%$ & & $90.6 \%$ WOSI & $93.5 \%$ & 76.4 \\
\hline Boileau et al.[17] & $17 \%$ & $2.1 \%$ & $0 \%$ & $17^{\circ} \mathrm{ER} 1$ & $\begin{array}{l}88 \mathrm{R} \\
87.6 \mathrm{~W}-\mathrm{D}\end{array}$ & & 16 \\
\hline Lafosse et al.[4] & $4 \%$ & $4 \%$ & $0 \%$ & $18^{\circ} \mathrm{ER}$ & & & 26 \\
\hline Castricini et al.[12] & $10 \%$ & $16.7 \%$ & $0 \%$ & $12^{\circ} \mathrm{ER}$ & $90 \mathrm{R}$ & & 13 \\
\hline Boileau et al.[19] & & $0 \%$ & $1.3 \%$ & & $\begin{array}{l}95 \mathrm{R} \\
96 \mathrm{~W}-\mathrm{D}\end{array}$ & $93 \%$ & 14 \\
\hline
\end{tabular}

ER1- external rotation loss in adduction, ER2- external rotation loss in abduction; R-Rowe, C- constant, W-D Walch-Duplay, SSV- subjective shoulder value; FU- follow-up (in months) 
of these patients had any signs of instability on clinical examination. An average follow-up in this group was significantly shorter and it might not allow the patients to fully appreciate the shoulder's stability. This thesis might be proven by the existing correlation between the time of the follow-up and the clinical results, which suggested that patients satisfaction improved with time. We have also developed two hypotheses regarding why patients after arthroscopic Latarjet stabilisation lost some external rotation in adduction to the side, more than in the open procedure:

1. subscapularis split using an ablation probe, more aggressive than gentle scissoring technique along muscular fibres, could create some scarring that limits rotation;

2. graft shape is slightly different: less decorticated on ventral side, less flat than in open procedure - this might cause mechanical impingement with subscapularis.

The parameters mentioned above: subjective apprehension and loss of external rotation in adduction to the side had a severe impact on the final clinical results.

\section{Radiographic results}

The rate of graft healing problems was significantly lower in ARTHRO than OPEN group: respectively three (5\%) and 11 $(26.2 \%)$. It is possible that arthroscopic technique could be less aggressive for a graft healing potential, however it also could be related to above mentioned surgeon's learning curve in open procedure. Shah et al. had $28 \%$ of non-unions in open stabilisation [16]. Butt and Charalambous reviewed the literature and reported $10.1 \%$ of nonunion and $1.6 \%$ of osteolysis after open procedure [25]. In arthroscopic technique the rate of graft healing problems varied from $7 \%$ : four non-unions and three graft osteolysis in the study of Lafosse and Boyle [4] to $22 \%$ reported by Casabianca et al. [29]. The opposite statement was found about partial graft osteolysis: it was significantly more frequent in ARTHRO than OPEN group: respectively $32(53.3 \%)$ versus ten $(23.8 \%)$. We consider this could be an effect of graft preparation and fixation whilst arthroscopic procedure, but this conclusion needs more investigation. Moroder et al. reported that bone block osteolysis could be a natural graft remodelling, depending on preoperative glenoid bone loss [30]. Also Di Giacomo et al. reported that osteolysis occured in the superficial and medial sections of the proximal part of the graft - this could be comparable to the results found in our study [31, 32].

\section{Conclusions}

For decades open Latarjet stabilisation has been proven to be a reliable method of anterior shoulder instability treatement
[13-16, 18, 21, 22], even in cases with large bony defects [33]. It could be concluded, that arthroscopic Latarjet procedure presents a good alternative for anterior shoulder instability treatement. The clinical results are comparable with the open procedure, as there was no statistically significant difference, but in three parameters: Rowe score, loss of external rotation with the arm at side and "subjective apprehension". Radiographic evaluation showed significantly lower rate of graft healing problems after arthroscopic stabilisation, however the partial osteolysis of the proximal part of the bone block was significantly more frequent. This study showed the arthroscopic technique results stay in proximity of the results after standard open procedure. The arthroscopic stabilisation is a relatively new technique requiring more detailed investigation. It is crucial to perform a well organised prospective study to achieve more reliable results. The conclusions drawn from these studies could lead to technical improvement to benefit from advantages of minimally invasive surgery.

Open Access This article is distributed under the terms of the Creative Commons Attribution 4.0 International License (http:// creativecommons.org/licenses/by/4.0/), which permits unrestricted use, distribution, and reproduction in any medium, provided you give appropriate credit to the original author(s) and the source, provide a link to the Creative Commons license, and indicate if changes were made.

\section{References}

1. Latarjet M (1954) Treatment of recurrent dislocation of the shoulder. Lyon Chir 49(8):994-997

2. Bhatia S, Frank RM, Ghodara NS et al (2014) The outcomes and surgical techniques of the Latarjet. Arthroscopy 30(2):227-235. doi:10.1016/j.arthro.2013.10.013

3. Walch G, Charret P, Pietro-Paoli H, Dejour H (1986) Anterior recurrent luxation of the shoulder. Postoperative recurrences. Rev Chir Orthop Reparatrice Appar Mot 72(8):541-555

4. Lafosse L, Boyle S (2010) Arthroscopic Latarjet procedure. J Shoulder Elb Surg 19(2):2-12. doi:10.1016/j.jse.2009.12.010

5. Rowe CR, Patel D, Southmayd WW (1978) The Bankart procedure: a long-term end-result study. J Bone Joint Surg Am 60(1):1-16

6. Wewers ME, Lowe NK (1990) A critical review of visual analogue scales in the measurement of clinical phenomena. Res Nurs Health 13:227-236

7. Yamamoto N, Itoi E, Abe H et al (2007) Contact between the glenoid and the humeral head in abduction, external rotation, and horizontal extension: a new concept of glenoid track. J Shoulder Elb Surg 16(5):649-656. doi:10.1016/j.jse.2006.12.012

8. Walch G, Young A (2012) Open Latarjet procedure for recurrent anterior instability of the shoulder. Operative technique. Personal experience. Aspetar Sports Med J 1(1):30-37

9. Lafosse L, Lejeune E, Bouchard A et al (2007) The arthroscopic Latarjet procedure for the treatement of anterior shoulder instability. Arthroscopy 23(11):1-5. doi:10.1016/j.arthro.2007.06.008

10. Griesser MJ, Harris JD, Mccoy BW et al (2013) Complications and reoperations after Bristow-Latarjet shoulder stabilization: a systematic review. J Shoulder Elb Surg 22(2):286-292. doi:10.1016/j. jse.2012.09.009 
11. Cunningham G, Benchouk S, Kherad O, Lädermann A (2016) Comparison of arthroscopic and open Latarjet with a learning curve analysis. Knee Surg Sports Traumatol Arthrosc 24(2):540-545. doi:10.1007/s00167-015-3910-3

12. Castricini R, De Benedetto M, Orlando N, Rocchi M, Zini R, Pirani P (2013) Arthroscopic Latarjet procedure: analysis of the learning curve. Musculoskelet Surg 97(1):93-98. doi:10.1007/s12306-0130262-3

13. Allain J, Goutallier D, Glorion C (1998) Long-term results of the Latarjet procedure for the treatment of anterior instability of the shoulder. J Bone Joint Surg Am 80:841-52

14. Mahirogullari M, Kuskucu M, Solakoglu C, Akmaz I, Pehlivan O, Kiral A (2006) Comparison of outcomes of two different surgeries in regarding to complications for chronic anterior shoulder instability. Arch Orthop Trauma Surg 126:674-679. doi:10.1007/s00402006-0190-x

15. Burkhart SS, De Beer JF, Barth JRH, Criswell T, Roberts C, Richards DP (2007) Results of modified Latarjet reconstruction in patients with anteroinferior instability and significant bone loss. Arthroscopy 23(10):1033-1041. doi:10.1016/j.arthro.2007.08.009

16. Shah AA, Butler RB, Romanowski J, Goel D, Karadagli D, Warner JJP (2012) Short-term complications of the Latarjet procedure. J Bone Joint Surg Am 94:495-501

17. Boileau P, Mercier N, Roussanne Y, C-édouard T, Old J (2010) Arthroscopic Bankart-Bristow-Latarjet procedure: the development and early results of a safe and reproducible technique. Arthroscopy 26:1434-1450

18. Hovelius L (2001) One hundred eighteen Bristow-Latarjet repairs for recurrent anterior dislocation of the shoulder prospectively followed for fifteen years: Study I - clinical results. J Shoulder Elb Surg 13(5):509-516. doi:10.1016/j.jse.2004.02.013

19. Boileau P, Gendre P, Baba M et al (2016) A guided surgical approach and novel fixation method for arthroscopic Latarjet. $\mathrm{J}$ Shoulder Elbow Surg 25(1):78-89. doi:10.1016/j.jse.2015.06.001

20. Dumont GD, Fogerty S, Rosso C, Lafosse L (2014) The Arthroscopic Latarjet procedure for anterior shoulder instability. Am J Sport Med 42(11):2560-6. doi:10.1177/0363546514544682

21. Cerciello S, Edwards TB, Walch G (2012) Chronic anterior glenohumeral instability in soccer players: results for a series of 28 shoulders treated with the Latarjet procedure. J Orthopaed Traumatol 13(4):197-202. doi:10.1007/s10195-012-0201-3

22. Mizuno N, Denard PJ, Raiss P, Melis B, Walch G (2014) Long-term results of the Latarjet procedure for anterior instability of the shoulder. J Shoulder Elbow Surg 23(11):1691-9. doi:10.1016/j. jse.2014.02.015

23. Ladermann A, Lubbeke A, Stern R, Cunningham G, Bellotti V, Gazielly DF (2013) Risk factors for dislocation arthropathy after
Latarjet procedure: a long-term study. Int Orthop 37:1093-1098. doi:10.1007/s00264-013-1848-y

24. Bessiere C, Trojani C, Pelegri C, Carles C, Carles M, Boileau P (2013) Coracoid bone block versus arthroscopic Bankart repair: a comparative paired study with 5-year follow-up. Orthop Traumatol Surg Res 99(2):123-30. doi:10.1016/j.otsr.2012.12.010

25. Butt U, Charalambous CP (2012) Complications associated with open coracoid transfer procedures for shoulder instability. J Shoulder Elbow Surg 21(8):1110-9

26. Collin P, Rochcongar P, Thomazeau H (2007) Treatment of chronic anterior shoulder instability using a coracoid bone block (Latarjet procedure): 74 cases. Rev Chir Orthop Reparatrice Appar Mot 93: 126-132 (in French)

27. Gohlke F, Janssen E, Leidel J, Heppelmann B, Eulert J (1998) Histopathological findings in the proprioception of the shoulder joint. Orthopade 27(8):510-7

28. Backenkohler U, Strasmann TJ, Halata Z (1997) Topography of mechanoreceptors in the shoulder joint region - a computer-aided $3 \mathrm{D}$ reconstruction in the laboratory mouse. Anat Rec 248(3):433-41

29. Casabianca L, Gerometta A, Massein A, Khiami F, Rousseau R, Hardy A, Pascal-Moussellard H, Loriaut P (2016) Graft position and fusion rate following arthroscopic Latarjet. Knee Surg Sports Traumatol Arthrosc 24(2):507-12. doi:10.1007/s00167-015-3551-6

30. Moroder P, Blocher M, Auffarth A, Hoffelner T, Hitzl W, Tauber M, Resch H (2014) Clinical and computed tomography-radiologic outcome after bony glenoid augmentation in recurrent anterior shoulder instability without significant glenoid bone loss. J Shoulder Elbow Surg 23(3):420-6. doi:10.1016/j.jse.2013.07.048

31. Giacomo GD, Costantini A, de Gasperis N, De Vita A, Lin BK, Francone M, Beccaglia MA, Mastantuono M (2011) Coracoid graft osteolysis after the Latarjet procedure for anteroinferior shoulder instability: a computer tomography scan study of twenty-six patients. J Shoulder Elbow Surg 20(6):989-995

32. Giacomo GD, de Gasperis N, Costantini A, De Vita A, Beccaglia MA, Pouliart N (2014) Does the presence of glenoid bone loss influence coracoid bone graft osteolysis after the Latarjet procedure? A computed tomography scan study in 2 groups of patients with and without glenoid bone loss. J Shoulder Elbow Surg 23(4): 514-518

33. Paladini P, Singla R, Merolla G, Porcellini G (2016) Latarjet procedure: is the coracoid enough to restore the glenoid surface? Int Orthop 40:1675-1681. doi:10.1007/s00264-015-3093-Z

34. Walch G (1987) Directions for the use of the quotation of anterior instabilities of the shoulder. Abstracts of the First Open Congress of the European Society of Surgery of the Shoulder and Elbow. Paris, $51-55$ 